\title{
EISENSTEIN SERIES TWISTED BY MODULAR SYMBOLS FOR THE GROUP $\mathrm{SL}_{n}$
}

\author{
Dorian Goldfeld and Paul E. Gunnells
}

\begin{abstract}
We define Eisenstein series twisted by modular symbols for the group $\mathrm{SL}_{n}$, generalizing a construction of the first author $[1,2]$. We show that, in the case of series attached to the minimal parabolic subgroup, our series converges for all points in a suitable cone. We conclude with examples for $\mathrm{SL}_{2}$ and $\mathrm{SL}_{3}$.
\end{abstract}

\section{Introduction}

1.1. Let $\Gamma$ denote a finitely generated discrete subgroup of $\mathrm{SL}_{2}(\mathbb{R})$ that contains translations and acts on the upper halfplane $\mathfrak{h}$. An automorphic form of real weight $r$ and multiplier $\psi: \Gamma \rightarrow \mathbf{U}$ (here $\mathbf{U}=\{w \in \mathbb{C}|| w \mid=1\}$ is the unit circle) is a meromorphic function $G: \mathfrak{h} \rightarrow \mathbb{C}$ that satisfies

$$
G(\gamma z)=\psi(\gamma) \cdot j(\gamma, z)^{r} \cdot G(z)
$$

for all $\gamma=\left(\begin{array}{ll}a & b \\ c & d\end{array}\right) \in \Gamma$ with $j(\gamma, z)=c z+d$. For $r \geq 0$, an integer, and $F: \mathfrak{h} \rightarrow \mathbb{C}$ any function with sufficiently many derivatives, G. Bol [3] proved the identity

$$
\frac{d^{r+1}}{d z^{r+1}}\left((c z+d)^{r} F(\gamma z)\right)=(c z+d)^{-r-2} F^{(r+1)}(\gamma z)
$$

which holds for all $\gamma \in \mathrm{SL}_{2}(\mathbb{R})$. It follows that if $f(z)$ is an automorphic form of weight $r+2$ and multiplier $\psi$, and if $F$ is any $(r+1)$-fold indefinite integral of $f$, then $F$ satisfies the functional equation

$$
F(\gamma z)=\psi(\gamma)(c z+d)^{-r}(F(z)+\phi(\gamma, z)),
$$

where $\phi(\gamma, z)$ is a polynomial in $z$ of degree $\leq r$ satisfying the cocycle condition

$$
\phi\left(\gamma_{1} \gamma_{2}, z\right)=\overline{\psi\left(\gamma_{2}\right)} j\left(\gamma_{2}, z\right)^{r} \phi\left(\gamma_{1}, \gamma_{2} z\right)+\phi\left(\gamma_{1}, z\right) .
$$

Such a function $F$ is called an automorphic (or Eichler) integral, and the corresponding polynomial $\phi(\gamma, z)$ is called a period polynomial.

Received April 24, 2000.

Revised October 1, 2000.

Both authors were partially supported by the NSF. 
1.2. The Eisenstein series $E^{*}(z ; r, \psi, \phi)$ (twisted by a period polynomial $\phi$ ) is defined by the infinite series

$$
E^{*}(z ; r, \psi, \phi)=\sum_{\gamma \in \Gamma_{\infty} \backslash \Gamma} \psi(\gamma) \phi(\gamma, z) j(\gamma, z)^{-r}
$$

The twisted Eisenstein series $E^{*}(z ; r, \psi, \phi)$ was first introduced by Eichler [4] (1965). Automorphic integrals and Eisenstein series twisted by period polynomials were systematically studied by Knopp [5] (1974). More recently [1, 2] (1995) nonholomorphic Eisenstein series twisted by modular symbols (period polynomials of degree 0) were introduced (cf. §5.3). O'Sullivan [6] found (using Selberg's method) the functional equation of these twisted Eisenstein series; very recently O'Sullivan and Chinta [7] explicitly computed the scattering matrix occurring in the functional equation.

In this paper we show how to generalize the construction of Eisenstein series twisted by modular symbols to the group $\mathrm{SL}_{n}$. The basic properties and region of absolute convergence of such series are obtained in the case of the minimal parabolic subgroup. We conjecture that these series satisfy functional equations.

\section{Eisenstein series}

2.1. In this section we recall the definition of cuspidal Eisenstein series following Langlands [8, Ch. 4]. We begin with some notation.

Let $G=\mathrm{SL}_{n}(\mathbb{R})$, let $K=\mathrm{SO}_{n}(\mathbb{R})$, and let $\Gamma \subset G(\mathbb{Z})$ be an arithmetic group. Let $P_{0} \subset G$ be the subgroup of upper-triangular matrices, and let $A_{0} \subset P_{0}$ be the subgroup of diagonal matrices with each entry positive. For each decomposition $n=n_{1}+\cdots+n_{k}$ with $n_{i}>0$, we have a standard parabolic subgroup

$$
P=\left\{\left(\begin{array}{ccc}
P_{1} & \cdots & * \\
& \ddots & \vdots \\
0 & & P_{k}
\end{array}\right) \mid P_{i} \in \mathrm{GL}_{n_{i}}(\mathbb{R}), \prod \operatorname{det}\left(P_{i}\right)=1\right\} .
$$

We fix a standard Langlands decomposition $P=M_{P} A_{P} N_{P}$ as follows: $M_{P}$ is the subgroup of block diagonal matrices, with each block an element of $\mathrm{SL}_{n_{i}}^{ \pm}(\mathbb{R})$; $A_{P} \subset P$ is the subgroup with the $i$ th block of the form $a_{i} I_{n_{i}}$, where $a_{i}>0$ and $I_{n_{i}}$ is the $n_{i} \times n_{i}$ identity; and $N_{P} \subset P$ is the subgroup with the $i$ th block equal to $I_{n_{i}}$. We transfer these decompositions to all rational parabolic subgroups by conjugation.

2.2. Let $\mathfrak{a}_{0}, \mathfrak{a}_{P}$ be the Lie algebras of the groups $A_{0}, A_{P}$. Let $\check{\mathfrak{a}}_{0}, \check{\mathfrak{a}}_{P}$ be their $\mathbb{R}$-duals, and denote the pairing by $\langle$,$\rangle . Let R=R^{+} \cup R^{-} \subset \check{\mathfrak{a}}_{0}$ be the roots of $G$, and let $\Delta \subset R^{+}$be the standard set of simple roots. For any root $\alpha$, let $\check{\alpha}$ be the corresponding coroot. For any parabolic subgroup $P$, let $\rho_{P}=1 / 2 \sum_{\alpha \in R^{+} \cap \check{\mathfrak{a}}_{P}} \alpha$.

We recall the definition of the height function $H_{P}: P \rightarrow \mathfrak{a}_{P}$. Given $p \in P$, write $p=\operatorname{man}$, where $m \in M_{P}, a \in A_{P}$, and $n \in N_{P}$. Then $H_{P}(p)$ is defined 
via

$$
e^{\left\langle\chi, H_{P}(p)\right\rangle}=a^{\chi}, \quad \text { for all } \chi \in \check{\mathfrak{a}}_{P} .
$$

Using an Iwasawa decomposition $G=P K$, we extend the height function to a map $H_{P}: G \rightarrow \mathfrak{a}_{P}$ by setting $H_{P}(g)=H_{P}(p)$, where $g=p k, p \in P, k \in K$.

2.3. Fix a parabolic subgroup $P$, and let $\Gamma_{P}=\Gamma \cap P$. Let $f \in C^{\infty}\left(A_{P} N_{P} \backslash G\right)$ be a $\Gamma_{P}$-invariant, $K$-finite function such that for each $g \in G$, the function $m \mapsto f(m g), m \in M_{P}$, is a square-integrable automorphic form on $M_{P}$ with respect to $\Gamma_{P} \cap M_{P}$. Let $\lambda \in\left(\check{\mathfrak{a}}_{P}\right) \otimes \mathbb{C}$, and let $g \in G$.

Definition 2.4. The Eisenstein series associated to the above data is

$$
E_{P}(f, \lambda, g)=\sum_{\gamma \in \Gamma_{P} \backslash \Gamma} e^{\left\langle\rho_{P}+\lambda, H_{P}(\gamma g)\right\rangle} f(\gamma g) .
$$

It is known [8, Lemma 4.1] that this series converges absolutely and uniformly on compact subsets of $G \times C$, where

$$
C=\left\{\lambda \mid\langle\Re \lambda, \check{\alpha}\rangle>\left\langle\rho_{P}, \check{\alpha}\right\rangle, \text { for all } \alpha \in \Delta\right\} ;
$$

here $\Re$ denotes real part.

\section{Modular symbols}

3.1. We recall the definition of modular symbols. Our definition is equivalent to that of Ash [9] and Ash-Borel [10], but we need a slightly different formulation for our purposes.

Let $V=\mathbb{Q}^{n}$ with the canonical $G(\mathbb{Q})$-action. Let w be a tuple of subspaces $\left(W_{1}, \ldots, W_{k}\right)$, where $W_{i} \subset V$. The type of $\mathbf{w}$ is the tuple $\left(\operatorname{dim} W_{1}, \ldots, \operatorname{dim} W_{k}\right)$. The tuple $\mathbf{w}$ is called full if $\sum \operatorname{dim} W_{i}=n$, and is called a splitting if $V=\bigoplus_{i} W_{i}$. Any splitting determines a rational flag

$$
F_{\mathbf{w}}=\left\{\{0\} \subsetneq F_{1} \subsetneq \cdots \subsetneq F_{k} \subsetneq V\right\}
$$

by $F_{j}=\bigoplus_{i \leq j} W_{i}$, and thus determines a rational parabolic subgroup $P_{\mathbf{w}}$, the stabilizer of $\bar{F}_{\mathbf{w}}$. We let $P_{\mathbf{w}}=M_{\mathbf{w}} A_{\mathbf{w}} N_{\mathbf{w}}$ be a choice of Langlands decomposition such that $M_{\mathbf{w}}(\mathbb{Q}) A_{\mathbf{w}}(\mathbb{Q})$ preserves each $W_{i}$. Note that this is not the same as the standard Langlands decomposition from $\S 2.1$.

3.2. Let $X$ be the symmetric space $G / K$, and let $\bar{X}$ be the bordification of $X$ constructed by Borel-Serre [11]. Then the cohomology $H^{i}(\Gamma ; \mathbb{C})$ may be identified with $H^{i}(\Gamma \backslash X ; \mathbb{C})$ and $H^{i}(\Gamma \backslash \bar{X} ; \mathbb{C})$.

Let $Y=\Gamma \backslash X, \bar{Y}=\Gamma \backslash \bar{X}, \partial \bar{Y}=\bar{Y} \backslash Y$, and let $\pi: X \rightarrow Y$ be the canonical projection. Let $d=\left(n^{2}+n\right) / 2-1$ be the dimension of $Y$. For all $i$, Lefschetz duality gives an isomorphism

$$
H_{d-i}(\bar{Y}, \partial \bar{Y} ; \mathbb{C}) \longrightarrow H^{i}(\Gamma ; \mathbb{C})
$$


3.3. Let $\mathbf{w}$ be a splitting, and let $K_{\mathbf{w}}$ be $K \cap M_{\mathbf{w}} A_{\mathbf{w}}$. The inclusion $M_{\mathbf{w}} A_{\mathbf{w}} \rightarrow$ $G$ induces a proper map

$$
\iota: M_{\mathbf{w}} A_{\mathbf{w}} / K_{\mathbf{w}} \longrightarrow X .
$$

Let $Y_{\mathbf{w}}$ be the closure of $(\pi \circ \iota)\left(M_{\mathbf{w}} A_{\mathbf{w}} / K_{\mathbf{w}}\right)$, and let $d(\mathbf{w})$ be the dimension of $Y_{\mathbf{w}}$. The submanifold $Y_{\mathbf{w}}$ is independent of the choice of decomposition in §3.1.

Definition 3.4. Let $\mathbf{w}=\left(W_{1}, \ldots, W_{k}\right)$ be a full tuple of subspaces. Then the modular symbol $\Xi_{\mathbf{w}}$ associated to $\mathbf{w}$ is defined as follows:

1. If $\mathbf{w}$ is a splitting, then $\Xi_{\mathbf{w}} \in H_{d(\mathbf{w})}(\bar{Y}, \partial \bar{Y} ; \mathbb{C})$ is the fundamental class of $Y_{\mathrm{w}}$.

2. Otherwise, $\Xi_{\mathbf{w}}$ is defined to be $0 \in H_{d(\mathbf{w})}(\bar{Y}, \partial \bar{Y} ; \mathbb{C})$, where $d(\mathbf{w})$ is the homological degree determined by any splitting with the same type as $\mathbf{w}$.

3.5. We define a $G(\mathbb{Q})$-action on tuples as follows. Given a full tuple $\mathbf{w}=$ $\left(W_{1}, \ldots, W_{k}\right)$, let $g \cdot \mathbf{w}$ be the tuple $\left(W_{1}, g W_{2}, \ldots, g W_{k}\right)$. By abuse of notation we write $g \cdot \Xi_{\mathbf{w}}$ for the modular symbol $\Xi_{g \cdot \mathbf{w}}$.

Note that this is not a $G(\mathbb{Q})$-action on modular symbols, since associativity does not hold. However, the definition $g \cdot \Xi_{\mathbf{w}}$ will suffice for our construction.

Note also that $g \cdot \Xi_{\mathbf{w}}$ is different from the modular symbol obtained via the natural $G(\mathbb{Q})$-action defined by left translation of all subspaces in a tuple. In particular, let $\gamma \in \Gamma$, and let $\mathbf{w}^{\prime}=\left(\gamma W_{1}, \ldots, \gamma W_{k}\right)$. Then $\Xi_{\mathbf{w}}=\Xi_{\mathbf{w}^{\prime}}$, but $\Xi_{\mathbf{w}} \neq \gamma \cdot \Xi_{\mathbf{w}}$ in general.

\section{Eisenstein series twisted by modular symbols}

4.1. Let $\mathbf{w}=\left(W_{1}, \ldots, W_{k}\right)$ be a full tuple of subspaces, and let $P$ be a rational parabolic subgroup. We say that $P$ and $\mathbf{w}$ are compatible if the following conditions hold: there is a splitting $\mathbf{w}^{\prime}=\left(W_{1}^{\prime}, \ldots, W_{k}^{\prime}\right)$ such that $P=P_{\mathbf{w}^{\prime}}$, the types of $\mathbf{w}$ and $\mathbf{w}^{\prime}$ are equal, and $W_{1}=W_{1}^{\prime}$.

Fix a rational parabolic subgroup $P$ and a compatible splitting w. Let $f, \lambda$ be as in $\S 2.3$, and let $\varphi$ be a $\mathbb{C}$-valued linear form on $H_{d(\mathbf{w})}(\bar{Y}, \partial \bar{Y} ; \mathbb{C})$.

Definition 4.2. The twisted Eisenstein series associated to the above data is

$$
E_{P, \varphi}^{*}=E_{P, \varphi}^{*}(f, \lambda, g, \mathbf{w})=\sum_{\gamma \in \Gamma_{P} \backslash \Gamma} \varphi\left(\gamma \cdot \Xi_{\mathbf{w}}\right) e^{\left\langle\rho_{P}+\lambda, H_{P}(\gamma g)\right\rangle} f(\gamma g) .
$$

We refer to $\S 5$ for examples of this series, and for a comparison with the construction in $[1,2]$.

Proposition 4.3. The series in (2) is well-defined.

Proof. Let $\mathbf{w}=\left(W_{1}, \ldots, W_{k}\right)$ and let $\gamma \in \Gamma$. We need to show that the modular symbol $\gamma \cdot \Xi_{\mathbf{w}}$ depends only on the coset $\Gamma_{P} \gamma$.

First we assume $\gamma \cdot \mathbf{w}$ is a splitting. By the remarks at the end of $\S 3.5$, if $\gamma \in \Gamma$ and $\mathbf{w}^{\prime}=\left(\gamma W_{1}, \ldots, \gamma W_{k}\right)$ is the tuple obtained by left translation, then $\Xi_{\mathbf{w}}=\Xi_{\mathbf{w}^{\prime}}$. From this it follows that if $\gamma_{P} \in \Gamma_{P}$, then $\left(\gamma_{P} \gamma\right) \cdot \Xi_{\mathbf{w}}=\Xi_{\mathbf{w}}$. Indeed, 
$\gamma_{P} \cdot \Xi_{\mathbf{w}}=\Xi_{\mathbf{w}^{\prime \prime}}$, where $\mathbf{w}^{\prime \prime}=\left(\gamma_{P}^{-1} W_{1}, W_{2}, \ldots, W_{k}\right)$, and any element of $\Gamma_{P}$ preserves $W_{1}$.

Now assume that $\gamma \cdot \mathbf{w}$ isn't a splitting. There are two possibilities: (1) $\gamma W_{i} \cap \gamma W_{j} \neq\{0\}$ for some $i, j>1$; (2) $\gamma W_{i} \cap \gamma W_{j}=\{0\}$ for all $i, j>1$ and $W_{1} \cap \gamma W_{j} \neq\{0\}$ for some $j$. In the first case, we have $\gamma \cdot \Xi_{\mathbf{w}}=0$ for all $\gamma$, so the Eisenstein series is identically 0 . In the second case, we have $\left(\gamma_{P} \gamma\right) \cdot \Xi_{w}=0$ for all $\gamma_{P} \in \Gamma_{P}$, since left translation of the tuple $\gamma_{P} \gamma \cdot \mathbf{w}$ by $\gamma_{P}^{-1}$ preserves the incidence conditions satisfied by the $W_{i}$. This completes the proof.

4.4. For the rest of this note, we will assume that $P$ is the minimal parabolic subgroup $P_{0}$, and will take $f \equiv 1$. Although the functions $E_{P, \varphi}^{*}$ are not automorphic, a certain sum of them is.

Proposition 4.5. Let $W_{i}, i=0, \ldots, n$ be 1-dimensional subspaces of $V$, and let $\mathbf{w}(i)$ be the tuple $\left(W_{0}, \ldots, \hat{W}_{i}, \ldots, W_{n}\right)$, where $\hat{W}_{i}$ means delete $W_{i}$. Then

$$
\varphi\left(\Xi_{\mathbf{w}(0)}\right) E_{P}(f, \lambda, g)=\sum_{i=1}^{n}(-1)^{i+1} E_{P, \varphi}^{*}(f, \lambda, g, \mathbf{w}(i)) .
$$

Proof. First, the twisted series on the right are well-defined, since if $P$ and $\mathbf{w}$ are compatible then so are $P$ and $\mathbf{w}(i)$ for each $i \geq 1$. We have the following basic relation among modular symbols for the minimal parabolic subgroup, from $[12,13]$ :

$$
\Xi_{\mathbf{w}(0)}=\sum_{i=1}^{n}(-1)^{i+1} \Xi_{\mathbf{w}(i)} .
$$

Note that the relations in $[12,13]$ imply that this equality holds true in $H_{d(\mathbf{w})}\left(Y_{\mathbf{w}}, \partial Y_{\mathbf{w}} ; \mathbb{C}\right)$ for any collection of 1-dimensional rational subspaces $\left(W_{0}, \ldots, W_{n}\right)$, even with the possibility that some $\mathbf{w}(i)$ aren't splittings. The result follows immediately from Definition 4.2 and the fact that if $\mathbf{w}^{\prime}=\left(\gamma W_{1}, \ldots, \gamma W_{n}\right)$ with $\gamma \in \Gamma$, then $\Xi_{\mathbf{w}^{\prime}}=\Xi_{\mathbf{w}(0)}$

Theorem 4.6. Let $P$ be the minimal parabolic subgroup $P_{0}$, and let $\mathbf{w}$ be a compatible splitting. Let $\varphi$ be a linear form on $H_{n-1}(\bar{Y}, \partial \bar{Y} ; \mathbb{C})$. Then the series (4.2) converges uniformly on compact subsets of $G \times C$, where $C$ is the cone (1).

Proof. We begin by recalling some facts from the theory of modular symbols associated to the minimal parabolic subgroup. These facts are equivalent to results in [13], and are just reformulated in terms of tuples and splittings.

Let $\mathcal{W}$ be the set of all full tuples of 1-dimensional subspaces. We define a function \|\|$: \mathcal{W} \rightarrow \mathbb{Z}$ as follows. From each 1-dimensional subspace $W$, we choose and fix a primitive vector $v(W) \in \mathbb{Z}^{n}$. Then we set

$$
\|\mathbf{w}\|=\left|\operatorname{det}\left(v\left(W_{1}\right), \ldots, v\left(W_{n}\right)\right)\right| .
$$


Let $\mathcal{W}_{u} \subset \mathcal{W}$ be the subset of tuples for which $\|\mathbf{w}\|=1$. The set $\Gamma \backslash \mathcal{W}_{u}$ is finite, where $\Gamma$ acts by left translations. One can show that any modular symbol $\Xi_{\mathbf{w}}$ can be written as a sum

$$
\Xi_{\mathbf{w}}=\sum_{\mathbf{w}^{\prime} \in S} \Xi_{\mathbf{w}^{\prime}}
$$

where $S$ is a finite subset of $\mathcal{W}_{u}$ (depending on $\Xi_{\mathbf{w}}$ ). Moreover, the cardinality of $S$ is bounded by $p(\log \|\mathbf{w}\|)$, where $p$ is a polynomial depending only on $n$ [14].

Let $\gamma \in \Gamma$ and consider the modular symbol $\gamma \cdot \Xi_{\mathbf{w}}$. Since $\mathbf{w}$ is compatible with $P$, the space $W_{1}$ is the span of the first basis element of $V$. Let us assume for the moment that for $i>1, W_{i}$ is the span of the $i$ th standard basis element of $V$. This implies that $\|\gamma \cdot \mathbf{w}\|$ is the absolute value of the determinant of a fixed $(n-1) \times(n-1)$ minor of $\gamma$. Hence

$$
\|\gamma \cdot \mathbf{w}\|<\max \left\{\left|\gamma_{i j}\right|^{n-1} \mid 1 \leq i, j \leq n\right\},
$$

where the implied constant depends only on $n$.

Let $M(\gamma)$ be the right hand side of (4). It follows that there is a polynomial $p_{1}$, depending only on $n$, such that

$$
p(\log \|\gamma \cdot \mathbf{w}\|)<p_{1}(\log M(\gamma))
$$

Now consider the value $\varphi\left(\gamma \cdot \Xi_{\mathbf{w}}\right)$. Since $\Gamma \backslash \mathcal{W}_{u}$ is finite, there is a maximum value $\varphi_{\max }$ that $|\varphi|$ attains on this set. Writing $I(\gamma, \lambda)=\exp \left(\left\langle\rho_{P}+\lambda, H_{P}(\gamma g)\right\rangle\right)$, we have

$$
\sum_{\gamma \in \Gamma_{P} \backslash \Gamma}\left|\varphi\left(\gamma \cdot \Xi_{\mathbf{w}}\right) I(\gamma, \lambda)\right|<<\sum_{\gamma \in \Gamma_{P} \backslash \Gamma}\left|p_{1}(\log M(\gamma)) I(\gamma, \lambda)\right|,
$$

where the implied constant depends on $n$ and $\varphi_{\max }$. The right of (5) has the same convergence properties as the usual Eisenstein series, and so the proof is complete under our assumption on $\mathbf{w}$.

Now assume $W_{i}$ is a general 1-dimensional subspace of $V$ for $i>1$. Let $v\left(W_{i}\right)_{j}$ be the $j$ th coordinate of $v\left(W_{i}\right)$, and let

$$
M(\mathbf{w})=\max \left\{\left|v\left(W_{i}\right)_{j}\right| \mid 1 \leq i, j \leq n\right\} .
$$

Then

$$
\|\gamma \cdot \mathbf{w}\|<<M(\gamma)
$$

where the implied constant depends on $n$ and $M(\mathbf{w})$. The rest of the proof proceeds as above. 


\section{Examples}

5.1. In this section we continue to assume that $P$ is the minimal parabolic subgroup $P_{0}$. We begin by discussing the connection between the construction in this note and that of $[1,2]$.

Let $\ell$ be a positive integer, let $G=\mathrm{SL}_{2}(\mathbb{R})$, and let $\Gamma=\Gamma_{0}(\ell)$. The space $X=\mathrm{SL}_{2}(\mathbb{R}) / \mathrm{SO}_{2}(\mathbb{R})$ is the upper halfplane $\mathfrak{h}$, and we let $\mathfrak{h}^{*}=\mathfrak{h} \cup \mathbb{P}^{1}(\mathbb{Q})$ be the usual partial compactification obtained by adjoining cusps. Given a pair of cusps $\left(q_{1}, q_{2}\right)$, we can determine a full tuple $\left(W_{1}, W_{2}\right)$ by setting $W_{i}$ to be the subspace of $\mathbb{Q}^{2}$ corresponding to the point $q_{i} \in \mathbb{P}^{1}(\mathbb{Q})$. Slightly abusing notation, we denote the corresponding modular symbol by $\Xi\left(q_{1}, q_{2}\right)$.

5.2. We can construct an interesting linear form on the modular symbols as follows. Let $f$ be a fixed weight two holomorphic cuspform on $\Gamma$. Then we set

$$
\varphi\left(\Xi\left(q_{1}, q_{2}\right)\right)=-2 \pi i \int_{q_{1}}^{q_{2}} f(z) d z,
$$

where the integration is taken along the ideal geodesic from $q_{1}$ to $q_{2}$. Note that if $f$ is a newform, then $\varphi(\Xi(\infty, 0))$ is the special value $-L(1, f)$.

To compute the series $(2)$, let $\Gamma_{\infty}=\Gamma \cap P$, and let $\Im: \mathfrak{h} \rightarrow \mathbb{R}$ be the imaginary part. Let $\alpha \in \check{\mathfrak{a}}_{0}$ be the standard positive root, so that $\rho_{P}=\alpha / 2$. Write $\lambda=t \alpha$, where $t \in \mathbb{C}$. It is easy to check that $e^{\left\langle\lambda+\rho_{P}, H_{P}(g)\right\rangle}=\Im(z)^{t+1 / 2}$, where $z \in \mathfrak{h}$ is the point corresponding to $g$. Setting $\left(q_{1}, q_{2}\right)=(\infty, 0)$, we see that the corresponding tuple $\mathbf{w}$ is compatible with $P$. We obtain

$$
E_{P, \varphi}^{*}(\lambda, g, \mathbf{w})=E_{P, \varphi}^{*}(t, z, \mathbf{w})=\sum_{\gamma \in \Gamma_{\infty} \backslash \Gamma} \varphi\left(\gamma \cdot \Xi_{\mathbf{w}}\right) \Im(\gamma z)^{t+1 / 2}, \quad t \in \mathbb{C} .
$$

By Theorem 4.6, this converges for $\Re t>1 / 2$.

5.3. To relate this to $[1,2]$, we recall the pairing between classical modular symbols and cuspforms. One fixes a point $z_{0} \in \mathfrak{h}^{*}$, and defines a map

$$
\begin{aligned}
{[]_{f}: \Gamma } & \longrightarrow \mathbb{C} \\
& \gamma \longmapsto-2 \pi i \int_{z_{0}}^{\gamma z_{0}} f(z) d z .
\end{aligned}
$$

(In $[1,2]$, this map is written as $\gamma \mapsto\langle\gamma, f\rangle$.) One can show that this map is independent of $z_{0}$, vanishes on $\Gamma_{\infty}$, and satisfies

$$
\left[\gamma \gamma^{\prime}\right]_{f}=[\gamma]_{f}+\left[\gamma^{\prime}\right]_{f}, \quad \text { for } \gamma, \gamma^{\prime} \in \Gamma .
$$

. Then the series in $[1,2]$ is defined by

$$
E^{*}(z, s)=\sum_{\gamma \in \Gamma_{\infty} \backslash \Gamma}[\gamma]_{f} \Im(\gamma z)^{s}, \quad s \in \mathbb{C},
$$

which converges for $\Re s>1$. 
To compare this with (6), let $q_{1}=\infty$ and $q_{2}=z_{0}=0$, and put $s=t+1 / 2$. Since

$$
\int_{q_{1}}^{q_{2}} f+\int_{q_{2}}^{\gamma q_{2}} f=\int_{q_{1}}^{\gamma q_{2}} f
$$

we find

$$
\varphi\left(\Xi\left(q_{1}, q_{2}\right)\right) E(z, s)+E^{*}(z, s)=E_{P, \varphi}^{*}(s-1 / 2, z, \mathbf{w})
$$

where

$$
E(z, s)=\sum_{\gamma \in \Gamma_{\infty} \backslash \Gamma} \Im(\gamma z)^{s}
$$

is the classical nonholomorphic Eisenstein series.

In $[1,2]$ it is shown that $E^{*}$ is "automorphic up to a shift." Precisely, if $\gamma \in \Gamma$, then

$$
E^{*}(\gamma z, s)=E^{*}(z, s)-[\gamma]_{f} E(z, s)
$$

This is easily seen to be equivalent to Proposition 4.5 above.

5.4. Now let $G=\mathrm{SL}_{3}(\mathbb{R})$, and let $\Gamma=\Gamma_{0}(\ell)$. This is the arithmetic group defined to be the subgroup of $G(\mathbb{Z})$ consisting of matrices with bottom row congruent to $(0,0, *) \bmod \ell$. The symmetric space $X=\mathrm{SL}_{3}(\mathbb{R}) / \mathrm{SO}_{3}(\mathbb{R})$ is a 5 -dimensional smooth noncompact manifold, and our modular symbols live in $\mathrm{H}_{2}(\bar{Y}, \bar{\partial} Y ; \mathbb{C})$.

To construct an interesting linear form on these modular symbols, we may use elements of the cuspidal cohomology $H_{\text {cusp }}^{3}(\Gamma ; \mathbb{C})$. These are classes that, via the de Rham isomorphism, correspond to $\Gamma^{\prime}$-invariant differential forms $\omega=$ $\sum_{I} f_{I} d \omega_{I}$, where the coefficients are cusp forms and $\Gamma^{\prime} \subset \Gamma$ is a torsionfree subgroup of finite index. In this context, $H_{\text {cusp }}^{3}(\Gamma ; \mathbb{C})$ can alternatively be defined to be the kernel of the restriction map $H^{3}(\bar{Y} ; \mathbb{C}) \rightarrow H^{3}(\partial \bar{Y} ; \mathbb{C})$. We refer to $[15]$ for details.

5.5. To explicitly construct classes in $H_{\text {cusp }}^{3}(\Gamma ; \mathbb{C})$ that can be paired with modular symbols, we may use techniques of [16]. There it is shown that $H_{\text {cusp }}^{3}(\Gamma ; \mathbb{C})$ is isomorphic to a space $W(\Gamma)$ of functions $f: \mathbb{P}^{2}(\mathbb{Z} / \ell \mathbb{Z}) \rightarrow \mathbb{C}$ satisfying certain relations [16, Summary 3.23]. A modular symbol $\Xi_{\mathbf{w}}$ modulo $\Gamma$ gives rise to a point $p_{\mathbf{w}} \in \mathbb{P}^{2}(\mathbb{Z} / \ell \mathbb{Z})$ by taking the bottom row of the matrix $\left(v\left(W_{1}\right), v\left(W_{2}\right), v\left(W_{3}\right)\right)$ [16, Prop. 3.12]. Hence given an element $\alpha \in H_{\text {cusp }}^{3}(\Gamma ; \mathbb{C})$ corresponding to a function $f_{\alpha} \in W(\Gamma)$, we obtain a linear form by setting

$$
\varphi\left(\Xi_{\mathbf{w}}\right)=f_{\alpha}\left(p_{\mathbf{w}}\right)
$$

This linear form is induced from the intersection pairing

$$
H_{3}(\bar{Y}) \times H_{2}(\partial \bar{Y}) \longrightarrow \mathbb{C}
$$

we refer to [16, Prop. 3.24] for details. 
5.6. For an explicit example, we may take $\ell=53$. This is the first level for which the cuspidal cohomology is nonzero; one finds that $\operatorname{dim} H_{\text {cusp }}^{3}\left(\Gamma_{0}(53) ; \mathbb{C}\right)=$ 2. A sample element is given as a function in $W(\Gamma)$ in Table II of [16].

To compute $E_{P, \varphi}^{*}$, we may take $\alpha \in H_{\text {cusp }}^{3}\left(\Gamma_{0}(53) ; \mathbb{C}\right)$ to be a Hecke eigenclass. For a prime $p$ with $(p, 53)=1$, the local $L$-factor of the representation corresponding to $\alpha$ has the form

$$
\left(1-a_{p} p^{-s}+\bar{a}_{p} p^{1-2 s}-p^{3-3 s}\right)^{-1},
$$

where $s \in \mathbb{C}$ and $a_{p}$ is the eigenvalue of a certain Hecke operator. If we fix an algebraic integer $\rho$ satisfying $\rho^{2}=-11$, we find that for our Hecke eigenclass

$$
a_{2}=-2-\rho, \quad a_{3}=-1+\rho, \quad a_{5}=1, \quad a_{7}=-3, \quad \ldots
$$

If we represent $\alpha$ using a function $f \in W(\Gamma)$, and apply the formulæ in [17, Ch. $\mathrm{V}$ and VII], we can obtain a very explicit expression for $E_{P, \varphi}^{*}$.

In contrast to the $\mathrm{SL}_{2}$ case, the twisted Eisenstein series on $\mathrm{SL}_{3}$ isn't simply automorphic up to a shift. If we consider the relation in Proposition 4.5, we see that a certain sum of three twisted Eisenstein series is equal to an automorphic function.

\section{References}

[1] D. Goldfeld, The distribution of modular symbols, Number theory in progress, Vol. 2 (Zakopane-Kościelisko, 1997), de Gruyter, 1999, pp. 849-865.

[2] Zeta functions formed with modular symbols, Automorphic forms, automorphic representations, and arithmetic (Fort Worth, TX, 1996), Proc. Sympos. Pure Math. 66, Amer. Math. Soc., Providence, RI, 1999, pp. 111-121.

[3] G. Bol, Invarianten linearer differentialgleichungen, Abh. Math. Sem. Univ. Hamburg 16 (1949), 1-28.

[4] M. Eichler, Grenzkreisgruppen und kettenbruchartige Algorithmen, Acta Arith. 11 (1965), $169-180$.

[5] Marvin I. Knopp, Some new results on the Eichler cohomology of automorphic forms, Bull. Amer. Math. Soc. 80 (1974), 607-632.

[6] Cormac O'Sullivan, Properties of Eisenstein series formed with modular symbols, J. Reine Angew. Math. 518 (2000), 163-186.

[7] G. Chinta and D. Goldfeld, Grössencharakter L-functions of real quadratic fields twisted by modular symbols, Inv. Math., to appear.

[8] R. P. Langlands, On the functional equations satisfied by Eisenstein series, Lecture Notes in Mathematics, Vol. 544, Springer-Verlag, 1976, v+337.

[9] A. Ash, Nonminimal modular symbols for $G L(n)$, Invent. Math., 91 (1988), 483-491.

[10] A. Ash and A. Borel, Generalized modular symbols, Cohomology of arithmetic groups and automorphic forms (Luminy-Marseille, 1989), Lecture Notes in Math. 1447, Springer, Berlin, 1990, pp. 57-75.

[11] A. Borel and J.-P. Serre, Corners and arithmetic groups (Avec un appendice: Arrondissement des variétés à coins, par A. Douady et L. Hérault), Comment. Math. Helv. 48 (1973), 436-491.

[12] A. Ash, Unstable cohomology of $S L(n, \mathscr{O})$, J. Algebra 167 (1994), 330-342.

[13] A. Ash and L. Rudolph, The modular symbol and continued fractions in higher dimensions, Invent. Math. 55 (1979), 241-250. 
[14] A. I. Barvinok, A polynomial time algorithm for counting integral points in polyhedra when the dimension is fixed, Math. Oper. Res. 19 (1994), 769-779.

[15] R. Lee and J. Schwermer, Cohomology of arithmetic subgroups of $\mathrm{SL}_{3}$ at infinity, J. Reine Angew. Math. 330 (1982), 100-131.

[16] A. Ash, D. Grayson, and P. Green, Computations of cuspidal cohomology of congruence subgroups of $S L(3, \mathbb{Z})$, J. Number Theory 19 (1984), 412-436.

[17] D. Bump, Automorphic forms on $G L(3, \mathbb{R})$, Lecture Notes in Mathematics 1083, Springer-Verlag, 1984.

Department of Mathematics, Columbia University, New York, New York 10027.

E-mail address: goldfeld@columbia.edu

Department of Mathematics, Rutgers University, Newark, New Jersey 07102.

E-mail address: gunnells@andromeda.rutgers.edu 